\title{
Influência dos tratamentos de termorretificação e furfurilação em propriedades tecnológicas de uma conífera
}

\author{
Influence of thermal rectification and furfurylation \\ treatments on technological properties of a \\ conifer
}

\author{
Ezequiel Gallio ${ }^{1}$, Paula Zanatta ${ }^{1}$, Nidria Dias Cruz ${ }^{1}$, \\ Gustavo Spiering Zanol ${ }^{2}$,Henrique Römer Schulz ${ }^{2}$, \\ Darci Alberto Gatto ${ }^{1}$
}

\footnotetext{
${ }^{1}$ Programa de Pós-Graduação em Ciência e Engenharia de Materiais, Universidade Federal de Pelotas, Pelotas, Rio Grande do Sul, Brasil.

${ }^{2}$ Curso de Engenharia Industrial Madeireira, Universidade Federal de Pelotas, Pelotas, Rio Grande do Sul, Brasil. e-mail: egeng.florestal@gmail.com, paulazanatta236@gmail.com,nidria_cruz@hotmail.com,darcigatto@yahoo.com, gustavo-zanol@hotmail.com, henriqueschulz09@hotmail.com
}

\section{RESUMO}

O Pinus elliottii Engelm. possui uma vasta possibilidade de aplicações, porém, apresenta características indesejáveis às quais podem ser atenuadas com a adoção dos tratamentos de termorretificação e a furfurilação. Assim, este trabalho analisou a influência que os tratamentos de furfurilição e termorretificação exerceram em propriedades tecnológicas da madeira de Pinus elliottii. Para tanto, confeccionaram-se corpos de prova com dimensões de 15 × 15 × $250 \mathrm{~mm}$ (tangencial, radial e longitudinal), distribuídos igualmente em 5 tratamentos: controle, furfurilação com carga de $25 \%$ de álcool furfurílico (T F25\%) e termorretificação em diferentes temperaturas $\left(\mathrm{T} 180^{\circ} \mathrm{C}, \mathrm{T} 200^{\circ} \mathrm{C}\right.$ e T $\left.220^{\circ} \mathrm{C}\right)$. Avaliaram-se a variação da massa $(\Delta \mathrm{M})$ segundo adaptação da norma ASTM D 2017 (2005), à massa específica aparente ao teor de umidade de 12\% ( $\left.\rho_{12 \%}\right)$, os módulos de elasticidade (MOE) e ruptura (MOR) obtidos pelo ensaio de flexão estática conforme a norma ASTM D 143 (2014), e a variação colorimétrica da madeira em conformidade com as recomendações do sistema CIE-L*a*b*. Verificou-se que o tratamento com furfurol provocou: aumentos significativos na massa, $\rho_{12 \%}$, MOR, e causou o escurecimento da madeira, não afetando significativamente o MOE, em 5\% de probabilidade de erro. Os tratamentos de termorretificação afetaram significativamente a perda de massa, contudo a maior diferença foi observada no tratamento $\mathrm{T} 220^{\circ} \mathrm{C}$, o qual acarretou redução significativa no MOR em relação ao grupo controle, destacando-se que tal tratamento deixou a madeira com coloração semelhante aquela adquirida pela madeira tratada com furfurol. Conclui-se que ambos os tratamentos causaram o escurecimento da madeira de Pinus elliottii agregando assim valor estético, sendo que o tratamento à base de furfurol, com carga de $25 \%$, ocasionou melhora nas propriedades tecnológicas da madeira. Apesar dos tratamentos de termorretificação terem aumentado o MOE, geraram perda de massa significativa, principalmente nos tratamentos mais agressivos, comprometendo assim possíveis usos da madeira com finalidade estrutural.

Palavras-chave: Pinus elliottii, modificação de madeira; colorimetria; propriedades físicas e mecânicas.

\section{ABSTRACT}

The Pinus elliottii Engelm. has a wide range of applications, but it has undesirable characteristics that can be attenuated with the adoption of thermal rectification and furfurylation treatments. Thus, this work analyzed the influence of two treatments of modification, furfurilatyon and thermal rectification, in technological properties of Pinus elliottii Engelm. wood. For this, samples with dimensions of 15 x 15 x 250 mm (tangential, radial and longitudinal) were prepared, distributed equally among 5 treatments: control, furfurylation with $25 \%$ loading of furfuryl alcohol (T F25\%) and thermortification at different temperatures ( $\mathrm{T} 180{ }^{\circ} \mathrm{C}, \mathrm{T}$ $200^{\circ} \mathrm{C}$ and $\mathrm{T} 220^{\circ} \mathrm{C}$ ). It were evaluated the mass loss (PM) according to ASTM D 2017 (2005), specific gravity at $12 \%$ moisture content $\left(\rho_{12} \%\right)$, elastic modulus (MOE) and rupture (MOR) by performing the static bending test in accordance with ASTM D 143 (2014), and wood color variation in accordance with the CIE- 
$\mathrm{L} * \mathrm{a} * \mathrm{~b} *$ system recommendations. It was verified that the treatment with furfurol caused: significant increases in the mass, $\rho_{12 \%}$, MOR, and caused the wood darkening, not significantly affecting the MOE, in 5\% probability of error. The thermal rectification treatments significantly affected the mass loss, however the highest difference was observed in the $\mathrm{T} 220^{\circ} \mathrm{C}$ treatment, which caused a significant reduction on MOR in relation to the control group, highlighting that such treatment left the wood with coloration similar to that obtained by wood treated with furfurol. It's concluded that both treatments caused darkening of Pinus elliottii wood, thus adding aesthetic value, being that the furfurol treatment with a $25 \%$ load caused an improvement on technological properties of wood. In spite of the thermal rectification treatments increased the MOE, they generated significant mass loss, mainly in the more aggressive treatments, thus compromising possible uses of the wood with structural purpose.

Keywords: Pinus elliottii; wood modification; colorimetry; physical and mechanical properties.

\section{INTRODUÇÃO}

A madeira de Pinus elliottii Engelm. está disponível em grande volume no setor madeireiro. Sua madeira pode ser empregada para diversas finalidades, como celulose e papel, extração de resina, movelaria e construção civil [1]. Contudo, a madeira dessa espécie é caracterizada por possuir baixa durabilidade e estabilidade dimensional, cor pálida e susceptibilidade à deterioração por agentes xilófagos.

Para atenuar esses efeitos indesejáveis, a utilização de processos que modifiquem as propriedades tecnológicas da madeira, visando à conservação ou melhora destas, estão em constante evolução por parte dos pesquisadores. Dentre estes processos, a retificação térmica e furfurilação da madeira destacam-se devido aos padrões estéticos e tecnológicos que conferem às madeiras tratadas.

Nesse contexto, o processo de modificação por furfurilação consiste na impregnação de álcool furfurílico no interior da madeira mediante o uso de autoclave. O álcool furfurílico pode ser obtido a partir de resíduos de biomassa vegetal [2]. Os estudos relacionados a esse tratamento tiveram início na década de 60, e analisando os resultados obtidos pelas pesquisas, observa-se que o furfurol fornece benefícios promissores à madeira [3]. As melhorias através da impregnação de álcool furfurílico, conduzem a redução da umidade de equilíbrio e aumento da estabilidade dimensional da madeira, que é proporcional ao aumento de massa, elevando assim a durabilidade [4]. Esse tratamento também proporciona alteração em parâmetros relacionados às propriedades mecânicas [2, 5].

Já o tratamento da termorretificação, quando aplicado na madeira, degrada os componentes químicos macromoleculares da madeira, iniciando com as hemiceluloses, seguido da celulose e, por fim, da lignina [6], ocasionando a perda de massa da madeira.

A madeira tratada pelo calor possui novas características, como: melhoras na estabilidade dimensional e resistência biológica ao ataque de fungos xilófagos [7, 8], maior durabilidade em serviço [9], melhores propriedades térmicas [10], maior valor estético em razão de sua coloração [11], adquirindo assim características parecidas às madeiras tratadas por meio da furfurilação.

Portanto, esta semelhança torna a impregnação com furfurol promissora, pois pode proporcionar alta proteção aos agentes xilófagos, melhor estabilidade dimensional, aumento de massa e o escurecimento, podendo ser uma alternativa à modificação da madeira por termorretificação.

Diante do exposto, este trabalhou objetivou analisar a influência que os tratamentos de termorretificação com três faixas de temperaturas e com furfurol acarretaram em propriedades físicas, mecânicas e colorimétricas da madeira de Pinus elliottii Engelm.

\section{MATERIAIS E MÉTODOS}

\subsection{Material utilizado}

Utilizaram-se tábuas da espécie Pinus elliottii Engelm., com aproximadamente 20 anos de idade, provenientes de um povoamento homogêneo, localizado no município de Piratini, Rio Grande do Sul.

A partir das tábuas, foram confeccionados corpos de prova com dimensões de $15 \mathrm{~mm}$ x $15 \mathrm{~mm}$ x 250 $\mathrm{mm}$ (planos tangencial, radial e longitudinal, respectivamente), os quais ficaram acondicionados em câmara climatizada, ajustada com umidade relativa de $65 \%$ e temperatura de $20^{\circ} \mathrm{C}$, até que atingissem a estabilização da massa. Atingido o equilíbrio higroscópico (12\% de umidade), efetuaram-se os tratamentos de termorretificação e furfurilação. As dimensões e as massas das amostras foram obtidas com o uso de paquímetro digital e balança analítica, com precisões de $\pm 0,01 \mathrm{~mm} \mathrm{e} \pm 0,01 \mathrm{~g}$, respectivamente. 


\subsection{Modificação da madeira}

Para o tratamento de termorretificação, realizado em uma estufa laboratorial, os corpos de prova permaneceram expostos à temperaturas de $180^{\circ} \mathrm{C}\left(\mathrm{T} 180^{\circ}\right), 200^{\circ} \mathrm{C}\left(\mathrm{T} 200^{\circ}\right)$ e $220^{\circ} \mathrm{C}\left(\mathrm{T} 220^{\circ}\right)$ por 2 horas.

Já para a modificação da madeira por furfurilação empregou-se uma única carga de álcool furfurílico (T F25\%), composta por: 25\% de álcool furfurílico, $65 \%$ de etanol, $5 \%$ de água destilada e 5\% de ácido cítrico. Visando a impregnação da solução na madeira utilizou-se uma autoclave horizontal laboratorial, empregando-se o processo de vácuo-pressão, com pressão de $8 \mathrm{kgf} / \mathrm{cm}^{2}$ durante 90 minutos.

Finalizados os tratamentos, os corpos de prova tratados foram encaminhados para uma estufa laboratorial, ajustada à uma temperatura de $70{ }^{\circ} \mathrm{C}$, para que ocorresse etapa de polimerização da solução no interior da madeira, determinado pela massa constante.

\subsection{Caracterizações das madeiras tratadas}

Finalizados os tratamentos, as propriedades físicas avaliadas foram a massa específica aparente ao teor de umidade de $12 \%\left(\rho_{12 \%}\right)$ por meio da Equação 1, e a variação de massa $(\Delta \mathrm{M})$ conforme adaptação da Equação 2, descrita pela norma ASTM D 2017 [12].

$$
\begin{aligned}
& \rho_{12 \%}=M_{12 \%} / V_{12 \%} \\
& \Delta M=\left(\left(M_{l}-M_{F}\right) / M_{l}\right) * 100
\end{aligned}
$$

Em que: $\rho_{12 \%}-$ massa específica aparente ao teor de umidade de $12 \%\left(\mathrm{~g} . \mathrm{cm}^{-3}\right) ; \mathrm{M}_{12 \%}-$ massa do corpo de prova ao teor de umidade de $12 \%(\mathrm{~g}) ; \mathrm{V}_{12 \%}$ - volume do corpo de prova ao teor de umidade de $12 \%\left(\mathrm{~cm}^{3}\right)$; $\Delta \mathrm{M}$ - perda de massa do corpo de após o tratamento (\%); $\mathrm{M}_{\mathrm{I}}$ - massa do corpo de prova antes do tratamento $(\mathrm{g}) ; \mathrm{M}_{\mathrm{F}}$ - massa do corpo de prova após o tratamento $(\mathrm{g})$.

Por meio do ensaio de flexão estática, seguindo as recomendações da norma ASTM D 143 [13], obtiveram-se os módulos de elasticidade (MOE) e ruptura (MOR) das madeiras submetidas aos tratamentos. Para isso, utilizou-se uma máquina universal de ensaios mecânicos (EMIC), com sistema computadorizado de aquisição de dados, equipada com célula de carga de 3 toneladas.

Já a variação colorimétrica em função dos tratamentos foi determinada com a utilização de aparelho colorímetro Konica Minolta CR 400, ângulo de observação de $10^{\circ}$ e sistema CIE L*a*b*. Avaliaram-se a luminosidade $\left(\mathrm{L}^{*}\right)$, coordenada cromática verde-vermelho $\left(\mathrm{a}^{*}\right)$, a coordenada cromática azul-amarelo $\left(\mathrm{b}^{*}\right)$, a saturação da cor $\left(\mathrm{C}^{*}\right)$, o ângulo de tinta $\left(\mathrm{h}^{*}\right)$ e a variação total da cor $(\Delta \mathrm{E})$, sendo esta última determinada com a utilização das Equações 3, 4, 5 e 6.

$$
\begin{aligned}
& \Delta L^{*}=L_{P T}^{*}-L^{*} A T \\
& \Delta a^{*}=a^{*}{ }_{P T}-a^{*} A T \\
& \Delta b^{*}=b^{*}{ }_{P T}-b^{*} A T \\
& \Delta E=\sqrt{ }\left(\Delta L^{*}\right)^{2}+\left(\Delta a^{*}\right)^{2}+\left(\Delta b^{*}\right)^{2}
\end{aligned}
$$

Em que: $\Delta \mathrm{L}^{*}$ - variação da luminosidade; $\Delta \mathrm{a}^{*}$ - variação da coordenada cromática verde-vermelho; $\Delta \mathrm{b}^{*}$ - variação da coordenada cromática azul-amarelo; $\Delta \mathrm{E}$ - variação total da cor da madeira; $\mathrm{L}_{\mathrm{AT}}$, $\mathrm{a}^{*}{ }_{\mathrm{AT}}$, $\mathrm{b}^{*}{ }_{\mathrm{AT}}$ - valores de luminosidade, coordenada cromática verde-vermelho e coordenada cromática azul-amarelo, antes à aplicação dos tratamentos, respectivamente; $\mathrm{L}_{\mathrm{PT}}, \mathrm{a}_{\mathrm{PT}}, \mathrm{b}_{\mathrm{PT}}-$ valores de luminosidade, coordenada cromática verde-vermelho e coordenada cromática azul-amarelo, posterior à aplicação dos tratamentos, respectivamente.

\subsection{Análise estatística dos resultados}


Obtidos os resultados, e por meio do teste LSD de Fisher, realizou-se a análise da variância (ANOVA) visando comparar e verificar a existência de diferenças significativas entre os diferentes tratamentos, efetuando-se posteriormente à comparação das médias. Determinou-se a correlação de Pearson para analisar o grau de dependência entre variáveis de interesse no estudo. As análises estatísticas foram desenvolvidas utilizando probabilidade de erro de $5 \%$.

\section{RESULTADOS}

\subsection{Propriedades físicas}

Com base nos resultados obtidos percebe-se que o tratamento de furfurilação da madeira incrementou significativamente a massa em relação ao grupo controle, enquanto os tratamentos de termorretificação acarretaram perdas de massa (PM) significativas em função do aumento das faixas de temperatura (Tabela 1).

Tabela 1: Resumo estatístico e valores médios de variação da massa $(\Delta \mathrm{M})$ e massa específica aparente à $12 \%$ de umidade $\left(\rho_{12 \%}\right)$ para os tratamentos na madeira de Pinus elliottii Engelm.

\begin{tabular}{c|c|c}
\hline TRATAMENTO & $\Delta \mathbf{M}(\%)$ & $\boldsymbol{\rho}_{\mathbf{1 2} \%}\left(\mathbf{g} \cdot \mathbf{c m}^{-\mathbf{3}}\right)$ \\
\hline Controle & - & $0,607(0,011) \mathrm{b}$ \\
\hline $\mathrm{T} \mathrm{F} 25 \%$ & $-15,19(1,41) \mathrm{a}$ & $0,644(0,006) \mathrm{a}$ \\
\hline $\mathrm{T} 180^{\circ} \mathrm{C}$ & $10,53(0,17) \mathrm{b}$ & $0,552(0,031) \mathrm{c}$ \\
\hline $\mathrm{T} 200^{\circ} \mathrm{C}$ & $12,66(0,34) \mathrm{c}$ & $0,550(0,031) \mathrm{c}$ \\
\hline $\mathrm{T} 220^{\circ} \mathrm{C}$ & $15,99(1,02) \mathrm{d}$ & $0,527(0,025) \mathrm{d}$ \\
\hline $\mathrm{CV}(\%)$ & 181,42 & 8,31 \\
\hline $\mathrm{F}$ & $2.364,25 *$ & $31,54 *$ \\
\hline
\end{tabular}

Em que: valores positivos e negativos para a variação da massa $(\Delta \mathrm{M})$ indicam perda e ganho de massa respectivamente. Valores entre parênteses apresentam o desvio padrão do tratamento, e médias nas colunas seguidas por mesma letra não diferem entre si, conforme o teste LSD Fisher, em 5\% de probabilidade de erro; CV - coeficiente de variação; $*$ - significativo em $5 \%$ de probabilidade de erro pelo teste $\mathrm{F}(\mathrm{p} \leq 0,05)$.

Comportamento semelhante à PM pode ser notado na massa específica aparente $\left(\rho_{12}\right)$, com exceção aos tratamentos $\mathrm{T} 180^{\circ} \mathrm{C}$ e T $200^{\circ} \mathrm{C}$, os quais não apresentaram diferenças significativas nessa propriedade física. Convém salientar que o tratamento T F $25 \%$ incrementou em aproximadamente $6,09 \%$ a $\rho_{12} \%$, enquanto o $\mathrm{T} 220^{\circ} \mathrm{C}$ causou decréscimo aproximado de $13,18 \%$.

\subsection{Propriedades mecânicas}

Quantos aos módulos de ruptura (MOR) e de elasticidade (MOE), provenientes do ensaio de flexão estática (Tabela 2), foi observada a ocorrência de diferenças significativas entre os tratamentos somente para o MOR. O tratamento com temperatura mais agressiva $\left(\mathrm{T} 220^{\circ} \mathrm{C}\right)$ reduziu em $28,46 \%$ o MOR. Já os demais tratamentos ocasionaram aumento desse parâmetro tecnológico, destacando-se o T F25\%, o qual proporcionou um ganho de aproximadamente $38,45 \%$ na resistência à ruptura após o processo de furfurilação.

Tabela 2: Resumo estatístico e valores médios dos parâmetros relacionados ao ensaio mecânico de flexão estática para os tratamentos na madeira de Pinus elliottii Engelm.

\begin{tabular}{c|c|c}
\hline TRATAMENTO & MOR (MPa) & MOE (MPa) \\
\hline Controle & $86,06(13,62) \mathrm{b}$ & $9.658,99(1.941,19) \mathrm{a}$ \\
\hline $\mathrm{T} \mathrm{F} 25 \%$ & $119,15(6,98) \mathrm{a}$ & $12.849,50(716,86) \mathrm{a}$ \\
\hline $\mathrm{T} 180^{\circ} \mathrm{C}$ & $97,81(19,45) \mathrm{b}$ & $11.974,2(1.638,75) \mathrm{a}$ \\
\hline $\mathrm{T} 200^{\circ} \mathrm{C}$ & $88,36(19,85) \mathrm{b}$ & $11.286,3(2.011,37) \mathrm{a}$ \\
\hline $\mathrm{T} 220^{\circ} \mathrm{C}$ & $61,57(2,99) \mathrm{c}$ & $10.897,4(680,96) \mathrm{a}$ \\
\hline
\end{tabular}




\begin{tabular}{c|c|c}
\hline $\mathrm{CV}(\%)$ & 24,61 & 16,25 \\
\hline $\mathrm{F}$ & $14,61 *$ & $5,34^{\mathrm{ns}}$ \\
\hline
\end{tabular}

Em que valores entre parênteses apresentam o desvio padrão do tratamento, e médias nas colunas seguidas por mesma letra não diferem entre si, conforme o teste LSD Fisher, em 5\% de probabilidade de erro; CV - coeficiente de variação; * - significativo em $5 \%$ de probabilidade de erro pelo teste $\mathrm{F}(\mathrm{p} \leq 0,05) ;{ }^{\text {ns }}$ - não significativo em $5 \%$ de probabilidade de erro pelo teste $\mathrm{F}(\mathrm{p}>0,05)$; MOR - módulo de ruptura; MOR - módulo de elasticidade.

Quanto ao MOE, o qual não apresentou diferença significativa, observou-se que todos os tratamentos apresentaram aumento nesse parâmetro tecnológico em relação ao grupo controle: 33,01\% (T F25\%), 23,97\%

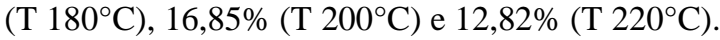

\subsection{Variação colorimétrica}

Percebe-se que os tratamentos de termorretificação e furfurilação provocaram escurecimento significativo da madeira (Figura 1 e Tabela 3) em comparação ao tratamento controle. Visualizam-se as tonalidades mais escuras nos tratamentos $\mathrm{T} \mathrm{F} 25 \%$ e T $220^{\circ} \mathrm{C}$, os quais não diferiram entre eles quanto aos parâmetros colorimétricos $\mathrm{L}^{*} \mathrm{e} \Delta \mathrm{E}$.

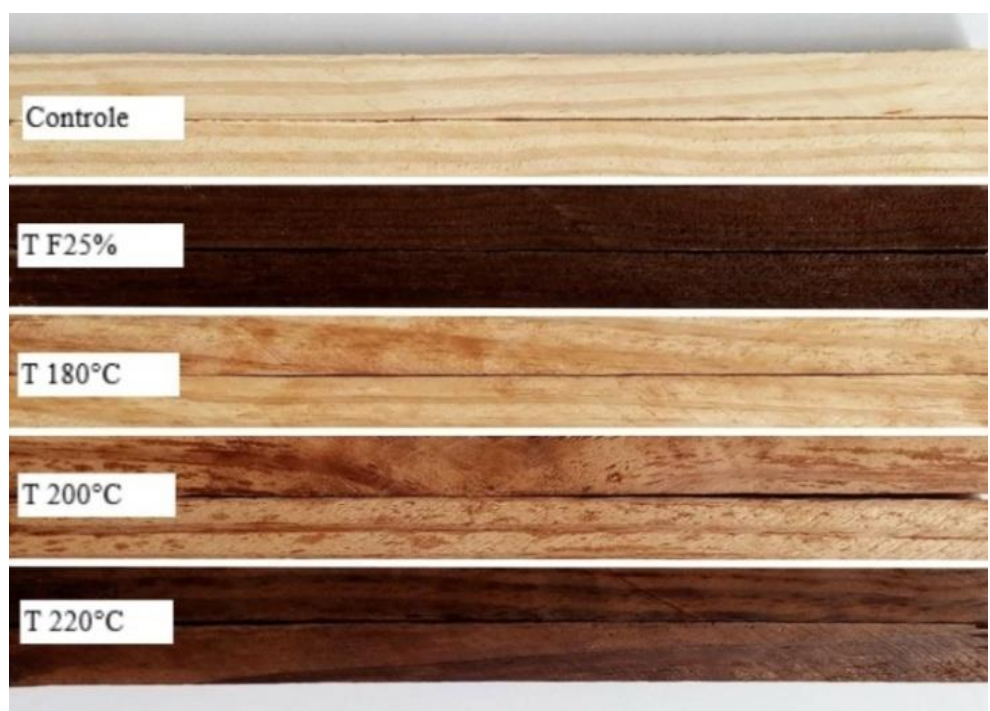

Figura 1: Variação da cor dos corpos de prova em função dos tratamentos aplicados.

No que tange aos parâmetros relacionados às coordenadas cromáticas $\mathrm{a}^{*} \mathrm{e} \mathrm{b}^{*}$, verifica-se que todos os tratamentos diferiram do tratamento controle. As maiores variações foram obtidas nos tratamentos $\mathrm{T} 200^{\circ} \mathrm{C}$, aumento de $102,59 \%$ para a coordenada cromática a*, e no $\mathrm{T} 220^{\circ} \mathrm{C}$, o qual reduziu a coordenada cromática b* em $42,35 \%$.

Tabela 3: Resumo estatístico e valores médios dos parâmetros colorimétricos para os tratamentos na madeira de Pinus elliottii Engelm.

\begin{tabular}{c|c|c|c|c|c|c}
\hline TRATAMENTOS & $\mathbf{L}^{*}$ & $\mathbf{a}^{*}$ & $\mathbf{b}^{*}$ & $\mathbf{C}^{*}$ & $\mathbf{h}$ & $\Delta \mathbf{E}$ \\
\hline Controle & $\begin{array}{c}76,77 \mathrm{a} \\
(5,92)\end{array}$ & $\begin{array}{c}5,41 \mathrm{a} \\
(1,27)\end{array}$ & $\begin{array}{c}24,44 \mathrm{~b} \\
(2,31)\end{array}$ & $\begin{array}{c}24,93 \mathrm{c} \\
(2,58)\end{array}$ & $\begin{array}{c}77,53 \mathrm{a} \\
(2,40)\end{array}$ & - \\
\hline $\mathrm{T} \mathrm{F} 25 \%$ & $\begin{array}{c}34,63 \mathrm{~d} \\
(2,39)\end{array}$ & $\begin{array}{c}9,51 \mathrm{~d} \\
(0,70)\end{array}$ & $\begin{array}{c}18,11 \mathrm{~d} \\
(1,88)\end{array}$ & $\begin{array}{c}20,47 \mathrm{~d} \\
(1,95)\end{array}$ & $\begin{array}{c}62,15 \mathrm{~d} \\
(1,43)\end{array}$ & $\begin{array}{c}42,68 \mathrm{a} \\
(6,84)\end{array}$ \\
\hline $\mathrm{T} 180^{\circ} \mathrm{C}$ & $\begin{array}{c}65,10 \mathrm{~b} \\
(6,70)\end{array}$ & $\begin{array}{c}8,22 \mathrm{~b} \\
(1,12)\end{array}$ & $\begin{array}{c}29,14 \mathrm{a} \\
(1,33)\end{array}$ & $\begin{array}{c}30,16 \mathrm{a} \\
(1,51)\end{array}$ & $\begin{array}{c}74,50 \mathrm{~b} \\
(1,80)\end{array}$ & $\begin{array}{c}15,03 \mathrm{c} \\
(5,26)\end{array}$ \\
\hline $\mathrm{T} 200^{\circ} \mathrm{C}$ & $\begin{array}{c}44,37 \mathrm{c} \\
(6,93)\end{array}$ & $\begin{array}{c}10,96 \mathrm{e} \\
(0,66)\end{array}$ & $\begin{array}{c}23,42 \mathrm{c} \\
(3,11)\end{array}$ & $\begin{array}{c}25,99 \mathrm{~b} \\
(2,81)\end{array}$ & $\begin{array}{c}64,72 \mathrm{c} \\
(2,84)\end{array}$ & $\begin{array}{c}33,77 \mathrm{~b} \\
(6,58)\end{array}$ \\
\hline
\end{tabular}




\begin{tabular}{c|c|c|c|c|c|c}
\hline $\mathrm{T} 220^{\circ} \mathrm{C}$ & $\begin{array}{c}34,55 \mathrm{~d} \\
(3,96)\end{array}$ & $\begin{array}{c}8,68 \mathrm{c} \\
(1,38)\end{array}$ & $\begin{array}{c}14,09 \mathrm{e} \\
(3,43)\end{array}$ & $\begin{array}{c}16,57 \mathrm{e} \\
(3,61)\end{array}$ & $\begin{array}{c}57,59 \mathrm{e} \\
(3,12)\end{array}$ & $\begin{array}{c}43,83 \mathrm{a} \\
(6,15)\end{array}$ \\
\hline $\mathrm{CV}(\%)$ & 34,96 & 24,84 & 26,72 & 22,76 & 11,81 & 39,07 \\
\hline $\mathrm{F}$ & $709,45 *$ & $209,82 *$ & $306,63 *$ & $239,5 *$ & $721,28 *$ & $272,74 *$ \\
\hline
\end{tabular}

Em que: valores entre parênteses apresentam o desvio padrão do tratamento, e médias nas colunas seguidas por mesma letra não diferem entre si, conforme o teste LSD Fisher, em 5\% de probabilidade de erro; CV - coeficiente de variação; * - significativo em $5 \%$ de probabilidade de erro pelo teste $\mathrm{F}(\mathrm{p} \leq 0,05)$; ns - não significativo em $5 \%$ de probabilidade de erro pelo teste $\mathrm{F}(\mathrm{p}>0,05)$; $\mathrm{L}^{*}$ - Luminosidade ou claridade; $\mathrm{a}^{*}$ - coordenada cromática verde-vermelho; $\mathrm{b}^{*}$ - coordenada cromática azul-amarelo; $\mathrm{C}^{*}$ - saturação da cor; $\mathrm{h}^{*}$ - ângulo de tinta; $\Delta \mathrm{E}$ - variação total da cor da madeira.

Outra variável colorimétrica que apresentou diferenças significativas dos demais tratamentos quando comparados ao grupo controle foi a $\mathrm{C}^{*}$, a qual demonstrou comportamento semelhante à coordenada cromática $b^{*}$, pois o tratamento $\mathrm{T} 220^{\circ} \mathrm{C}$ reduziu $33,53 \%$ em relação ao controle. Por fim, considerando o h, verifica-se que o $\mathrm{T} 220^{\circ} \mathrm{C}$ teve um decréscimo de aproximadamente $25,72 \%$ do valor encontrado para o tratamento controle. Para todas as variáveis colorimétricas analisadas, o tratamento $\mathrm{T} 180^{\circ} \mathrm{C}$ apresentou à menor variação de cor em comparação com o controle.

Por meio da análise das variáveis colorimétricas $\mathrm{L}^{*}, \mathrm{a}^{*}, \mathrm{~b}^{*}, \mathrm{C}$ e h, obtidas neste estudo e o agrupamento de Cluster considerando essas variáveis [14], foi possível determinar as cores que a madeira de P. elliottii adquiriu em função dos diferentes tratamentos aplicados: branco - controle; oliva - T F25\%; amareloclaro - T $180^{\circ} \mathrm{C}$; marrom-claro - T $200^{\circ} \mathrm{C}$ e marrom-escuro - T $220^{\circ} \mathrm{C}$.

\subsection{Correlação de Pearson}

Entre as variáveis analisadas, nota-se uma correlação significativa e inversa à PM, a $\rho 12 \%$ e o MOR (Tabela 4). Este último correlaciona-se de forma significativa com os parâmetros colorimétricos $b^{*}$ e $C^{*}$ e com o MOE.

Tabela 4: Correlação de Pearson relacionando características físicas, mecânicas e colorimétricas de interesse.

\begin{tabular}{c|c|c|c|c|c|c|c|c|c|c}
\hline & $\mathbf{P}_{\mathbf{1 2} \%}$ & $\mathbf{P M}$ & $\mathbf{M O R}$ & $\mathbf{M O E}$ & $\mathbf{L}^{*}$ & $\mathbf{a}^{*}$ & $\mathbf{b}^{*}$ & $\mathbf{C}^{*}$ & $\mathbf{h}$ & $\Delta \mathbf{E}$ \\
\hline $\mathbf{P}_{12 \%}$ & - & $-0,846$ & 0,635 & 0,257 & 0,084 & $-0,192$ & 0,015 & $-0,015$ & 0,196 & $-0,143$ \\
\hline $\mathbf{P M}$ & & - & $-0,566$ & $-0,073$ & $-0,005$ & 0,144 & 0,049 & 0,073 & $-0,128$ & 0,025 \\
\hline $\mathbf{M O R}$ & & & - & 0,675 & 0,084 & 0,145 & 0,342 & 0,360 & 0,219 & $-0,102$ \\
\hline $\mathbf{M O E}$ & & & & - & $-0,215$ & 0,353 & 0,061 & 0,106 & $-0,151$ & 0,207 \\
\hline $\mathbf{L}^{*}$ & & & & & - & $-0,726$ & 0,768 & 0,688 & 0,974 & $-0,966$ \\
\hline $\mathbf{a}^{*}$ & & & & & & - & $-0,167$ & $-0,043$ & $-0,652$ & 0,719 \\
\hline $\mathbf{b}^{*}$ & & & & & & & - & 0,992 & 0,838 & $-0,741$ \\
\hline $\mathbf{C}^{*}$ & & & & & & & & - & 0,766 & $-0,660$ \\
\hline $\mathbf{h}$ & & & & & & & & & - & $-0,954$ \\
\hline$\Delta \mathbf{E}$ & & & & & & & & & & - \\
\hline
\end{tabular}

Em que: valores de correlação destacados na cor vermelha são significativos, considerando 5\% de probabilidade de erro; $\rho_{12 \%}$ - massa específica aparente ao teor de umidade de equilíbrio de $12 \%\left(\mathrm{~g} / \mathrm{cm}^{3}\right) ; \mathrm{PM}$ - perda de massa $(\%)$; MOR módulo de ruptura (MPa); MOE - módulo de elasticidade $(\mathrm{MPa})$; L* - Luminosidade ou claridade; $\mathrm{a}^{*}$ - coordenada cromática verde-vermelho; $\mathrm{b}^{*}$ - coordenada cromática azul-amarelo; $\mathrm{C}^{*}$ - saturação da cor; $\mathrm{h}$ - ângulo de tinta; $\Delta \mathrm{E}-$ variação total da cor da madeira.

O MOE por sua vez correlacionou-se significativamente com o parâmetro colorimétrico a*. Observando os parâmetros colorimétricos, constata-se a inexistência de correlação significativa somente entre a coordenada cromática $\mathrm{a}^{*}$, com a coordenada cromática $\mathrm{b}^{*}$ e a saturação da cor $\mathrm{C}^{*}$.

\section{DISCUSSÃO}

O tratamento de furfurilação acarretou o ganho de massa devido ao processo de polimerização do furfurol no 
interior da madeira e fixação na parede celular desse material, corroborando com o relatado por MANTANIS; LIKYDIS [4]. Em estudo utilizando diferentes concentrações de furfurol na espécie Pinus massoniana, DONG et al. [15] obtiveram ganhos de massa de $33,92 \%, 49,44 \%$ e 55,63\% para as respectivas concentrações de $30 \%, 50 \%$ e $70 \%$, sendo estes superiores ao valor encontrado no presente estudo.

Quando se aplica o processo de termorretificação, quanto mais agressivas as temperaturas e o tempo empregados, maior é a taxa de degradação dos componentes químicos da madeira, principalmente as hemiceluloses e a celulose. Inicialmente, o decréscimo de massa pode ser atribuído à perda de umidade e componentes voláteis da madeira [16]. Acima de $100{ }^{\circ} \mathrm{C}$, por às hemiceluloses apresentarem maior instabilidade térmica $[17,18]$ quando comparadas à celulose e à lignina, explica o aumento progressivo da perda de massa em função da agressividade do tratamento de retificação térmica. Posteriormente, inicia-se a degradação mais intensa da celulose e na sequência da lignina.

POUBEL et al. [7] ao submeter a madeira de Pinus caribaea a tratamento de termorretificação por período de 2 horas e temperaturas de $200{ }^{\circ} \mathrm{C}$ e $220{ }^{\circ} \mathrm{C}$, obteve perdas de massa de $10,41 \%$ e $19,28 \%$, respectivamente, indicando comportamento semelhante ao encontrado neste estudo, no que tange a maior perda de massa como aumento da temperatura.

O aumento nos módulos de elasticidade (MOE) e ruptura (MOR) em função da impregnação de furfurol na madeira pode estar associado com o processo de polimerização in situ ocorrida nos lúmens e na parede celular. O álcool furfurílico, devido sua elevada polaridade, reage com a parede celular da madeira, polimerizando-se após a aplicação de temperaturas [4,19]. Assim, as modificações recorrentes na estrutura química da madeira em função do processo de polimerização podem contribuir para o enrijecimento da parede celular, e consequente aumento de suas propriedades mecânicas.

Mesmo que inicialmente as menores faixas de temperatura $\left(180^{\circ} \mathrm{C}\right.$ e $\left.200{ }^{\circ} \mathrm{C}\right)$ tenham aumentado os valores de MOE e MOR em comparação ao tratamento controle, obteve-se uma tendência de redução dessas propriedades em função do aumento da agressividade da termorretificação, conforme observado por CADEMARTORI et al. [10] em sua pesquisa. Conforme descrito anteriormente, por meio da aplicação de altas temperaturas durante o processo, tem-se a degradação dos polímeros que constituem o material. Altas temperaturas podem transformar celulose amorfa em cristalina [8], e que em conjunto com a redução do teor de umidade de equilíbrio da madeira, em função da redução da quantidade de grupos $\mathrm{OH}$-(hidroxilas), acarretaria em um aumento da resistência mecânica, corroborando o abordado por BOONSTRA et al. [20].Contudo, a degradação das hemiceluloses em função do aumento de temperatura pode contribuir para a redução da resistência mecânica, indo de acordo com o proposto por ESTEVES; PEREIRA [21].

A variável colorimétrica luminosidade $\left(\mathrm{L}^{*}\right)$ varia de 0 a 100, e representa as cores preta e branca, respectivamente [11]. O decréscimo desse valor do tratamento controle em comparação à madeira furfurilada pode ocorrer devido ao furfurol, que em sua forma líquida, apresenta uma coloração avermelhada. Há de se destacar que para o processo de polimerização aplicou-se uma temperatura de $70{ }^{\circ} \mathrm{C}$, a qual pode ter contribuído para o escurecimento. Pesquisas que destacam a polimerização da madeira com furfurol e sua influência nas características colorimétricas ainda são escassas. A redução da variável colorimétrica $L^{*}$ dos tratamentos de termorretificação em relação ao grupo controle é atribuída à degradação das hemicelulosese à alteração das características e migração dos extrativos dos extrativos $[10,22,23]$ tendência essa observada também por MOURA; BRITO [24] e PERTUZZATTI et al. [25].

A coordenada cromática $a^{*}$ (com sinal positivo $(+)$ representa que a madeira tende á tonalidade vermelha, enquanto que sinal negativo (-) antes de valor destaca que a madeira possui uma tendência a cor verde $[11,25]$. O aumento dos valores dessa variável em função dos tratamentos indica que houve modificação dos componentes químicos da madeira [26]. Para as madeiras termorretificadas, esta modificação é dada pelas degradações dos polímeros e para a madeira furfurilada pela polimerização. CADERMARTORI et al. [10], MOURA; BRITO [24] e PERTUZZATTI et al. [25] observaram comportamento semelhante ao do presente estudo em relação à variável cromática $\mathrm{a}^{*}$, isto é, há um aumento destes valores com a retificação térmica.

Já a coordenada cromática b* (matriz amarela) associa-se com a cor azul e amarela, quando representada pelos sinais negativo (-) e positivo (+), respectivamente [11, 25]. Devido à madeira de Pinus elliottii tender a um tom mais amarelado, é esperado que o tratamento de termorretificação ocasione a redução desse parâmetro, pois os extrativos e a lignina estão diretamente associados com cromóforos responsáveis pela coloração amarela [23], acarretando no escurecimento da madeira.

A variável colorimétrica saturação $\left(\mathrm{C}^{*}\right)$ indica a pureza da cor em relação ao branco, sendo que quanto menor for seu valor, mais opaca torna-se a cor da madeira [27]. Os tratamentos de termorretificação ocasionaram redução do valor de $\mathrm{C}^{*}$, demonstrando comportamento semelhante à coordenada cromática $\mathrm{b}^{*}$. Isto indica que uma vez que os valores de $b^{*}$ são maiores dos que o de $a^{*}$, a saturação $C^{*}$ tende a ser influenciada 
principalmente pela matriz amarela $\left(\mathrm{b}^{*}\right)$, estando isso em conformidade com o argumentado por ZANUNCIO et al. [11]. Tais reduções na saturação e sua associação com a matriz amarela, em função de tratamentos de termorretificação também foram observadas por PINCELLI et al. [23] e CADEMARTORI et al. [10].

$\mathrm{O}$ ângulo de tinta $\mathrm{h}$ caracteriza a predominância de uma determinada tonalidade em uma cor, aonde varia de $0^{\circ}$ a $90^{\circ}$, representando as cores vermelho e amarelo, respectivamente [23, 28]. Tal como no presente estudo, CADEMARTORI et al. [10], ZANUNCIO et al. [11] e PERTUZZATTI et al. [25] notaram a redução do ângulo de tinta de madeiras termorretificadas, indicando o escurecimento das mesmas, tendendo a uma tonalidade mais avermelhada.

\section{CONCLUSÕES}

A aplicação de todos os tratamentos térmicos e de furfurilação acarretou modificações nas propriedades físicas, mecânicas e colorimétricas analisadas da madeira de Pinus elliottii Engelm. Verificou-se que o tratamento à base de álcool furfurílico, com carga de $25 \%$, gera melhorias significativas na massa, MOE e MOR, além do proporcionar uma tonalidade mais escura, agregando valor estético à madeira tratada.

Dos tratamentos de termorretificação, a temperatura mais agressiva $\left(\mathrm{T} 220^{\circ} \mathrm{C}\right)$ diminuiu indesejavelmente e consideravelmente a massa e o MOR, entretanto, tal faixa de temperatura escureceu à madeira, deixando-a semelhante àquela submetida ao tratamento de furfurilação.

Do ponto de vista da qualidade da madeira, a utilização do furfurol apresenta-se como uma alternativa promissora aos tratamentos de madeira que visam melhores respostas quanto às propriedades tecnológicas inerentes ao material. Visto o potencial da furfurilação da madeira com estes resultados, recomendam-se mais estudos testando diferentes cargas de furfurol com distintas espécies madeireiras, a fim de determinar cargas eficientes em função da finalidade à qual se pretende empregar à madeira furfurilada.

\section{AGRADECIMENTOS}

À Coordenação de Aperfeiçoamento do Pessoal de Nível Superior (CAPES) pela concessão da bolsa de doutorado e ao Conselho Nacional de Desenvolvimento Científico e Tecnológico (CNPq).

\section{BIBLIOGRAFIA}

[1] FLORIANO, E. P., SCHENEIDER, P. R., FINGER, C. A., et al., "Análise econômica da produção de Pinus elliottii na Serra do Sudeste, Rio Grande do Sul”, Ciência Florestal, v. 19, n. 4, pp. 393-406, 2009.

[2] LANDE, S., WESTIN, M., SCHNEIDER, M., "Properties of furfurylated wood", Scandinavian Journal of Forest Research, v. 19, n. 5, pp. 22-30, 2004.

[3] HADI, Y.S., WESTIN, M., RASYID, E., "Resistance of furfurylated wood to termite attack", Forest Products Journal, v. 55, n. 11, pp. 85-88, 2005.

[4] MANTANIS, G., LYKIDIS, C., "Evaluation of weathering of furfurylated wood decks after a 3-year outdoor exposure in Greece", Drvna Industrija, v. 66, n. 2, pp. 115-122,2015.

[5] EPMEIER, H., WESTIN, M., RAPP, A., "Differently modified wood: comparison of some selected properties", Scandinavian Journal of Forest Research, v. 19, n. 5, pp. 31-37, 2004.

[6] GAŠPAROVIČ, L., LABOVSKÝ, J., MARKOŠ, J., et al., "Calculation of kinetic parameters of the thermal decomposition of wood by distributed activation energy model (DAEM)", Chemical and Biochemical Engineering Quarterly, v. 26, n. 1, p. 45-53, 2012.

[7] POUBEL, D. S., GARCIA, R. A., SANTOS, W. A., et al., "Efeito da termorretificação nas propriedades físicas e químicas da madeira de Pinus caribeae", Cerne, Lavras, v. 19, n. 3, pp. 391-398, 2013.

[8] CALONEGO, F. W., SEVERO, E. T. D., BALLARIN, A. W., "Physical and mechanical properties of thermally modified wood from E. grandis", European Journal of Wood and Wood Products, v. 70, n. 4, pp. 453-460, 2012.

[9] TREVISAN, H., LATORRACA, J. V. F., SANTOS, A. L. P., TEIXEIRA, J. G., CARVALHO. A. G., "Analysis of rigidity loss and deterioration from exposure in a decay test field of thermorectificated Eucalyptus grandis wood", Maderas. Ciencia y tecnologia, v. 16, n. 2, pp. 217-226, 2014.

[10] CADEMARTORI, P. H. G., MISSIO, A. L., MATTOS, B. D., SCHNEID, E., GATTO, D. A., "Physical and mechanical properties and colour changes of fast-growing Gympie messmate wood subjected to two-step steam-heat treatments", Wood Material Science and Engineering, v. 9, n. 1, pp. 40-48, 2014. 
[11] ZANUNCIO, A. J. V., FARIAS, E. S., SILVEIRA, T. A., "Termorretificação e colorimetria da madeira de Eucalyptus grandis", Floresta e Ambiente, v. 21, n.1, pp. 85-90, 2014.

[12] AMERICAN SOCIETY FOR TESTING AND MATERIALS (ASTM), "Standard test method of accelerated laboratory test of natural decay resistance of woods", ASTM D 2017, Annual Book of ASTM Standards, ASTM, West Conshohocken, PA, 2005.

[13] AMERICAN SOCIETY FOR TESTING AND MATERIALS (ASTM), "Standard test methods for small clear specimens of timber", ASTM D 143 - 94, Philadelphia, 2014.

[14] CAMARGOS, J. A. A., GONÇALEZ, J. C., "A colorimetria aplicada como instrumento na elaboração de uma tabela de cores de madeira", Brasil Florestal, n. 71, p. 30-41, pp. 679-690, 2007.

[15] DONG, Y., QIN, Y., WANG, K., et al., "Assessment of the performance of furfurylated wood and acetylated wood: comparison among four fast-growing wood species", BioResources, v. 11, n. 2, pp. 3679-3690, 2016.

[16] KRAUSS, A., PIERNIK, M., PINKOWSKI, G., "Cutting power during milling of thermally modified pine wood", Drvna Industrija, v. 67, n. 3, pp. 215-222, 2016.

[17] MODES, K. S., SANTINI, E. J., VIVIAN, M. A., et al., "Efeito da termorretificação nas propriedades mecânicas das madeiras de Pinus taeda e Eucalyptus grandis", Ciência Florestal, Santa Maria, v. 27, n. 1, pp. 291-302, 2017.

[18] ALFREDSEN, G., BADER, T. K., DIBDIAKOVA, J., et al., "Thermogravimetric analysis for wood decay characterization", European Journal of Wood and Wood Products, v. 70, n. 4, pp. 527-530, 2012.

[19] PILGÅRD, A., TREU, A., VAN ZEELAND, A. N. T., et al., "Toxic hazard and chemical analysis of leachates from furfurylated wood", Environmental Toxicology and Chemistry, v. 29, n. 9, pp. 1918-1924, 2010.

[20] BOONSTRA, M. J., VAN ACKER, J., TJEERDSMA, B. F., et al., "Strength properties of thermally modified softwoods and its relation to polymeric structural wood constituents", Annals of Forest Science, v. 64, n. 7, pp. 679-690, 2007.

[21] ESTEVES, B., PEREIRA, H. M., "Wood modification by heat treatment: a review", BioResources, v. 4, n. 1, pp. 370-404, 2009.

[22] DUBEY, M. K., PANG, S., WALKER, J., "Changes in chemistry, color, dimensional stability and fungal resistance of Pinus radiata D. Don wood with oil heat-treatment", Holzforschung, v. 66, pp. 49-57, 2012.

[23] PINCELLI, A. L. P. S. M., MOURA, L. F., BRITO, J. O., "Effect of thermal rectification on colors of Eucalyptus saligna and Pinus caribaea woods", Maderas. Ciencia y tecnología, v. 14, n. 2, pp. 239-248, 2012.

[24] MOURA, L. F., BRITO, J. O., "Efeito da termorretifcação sobre as propriedades colorimétricas das madeiras de Eucalyptus grandis e Pinus caribaea var. hondurensis", Scientia Forestalis, Piracicaba, v. 39, n. 89, pp. 69-76, 2011.

[25] PERTUZZATTI, A., MISSIO, A. L., CONTE, B., et al., "Propriedades físicas da madeira de Pinus elliottii var. elliottii termorretificada sob diferentes atmosferas", Ciência da Madeira, v. 7, n. 1, pp. 7-15, 2016.

[26] CHEN, Y., FAN, Y., GAO, J., et al., "The effect of heat treatment on the chemical and color change of black locust (Robinia pseudocacia) wood flour", BioResources, v. 7, n. 1, pp. 1157-1170, 2012.

[27] MATTOS, B. D., CADEMARTORI, P. H. G., LOURENÇON, T. V., et al., "Colour changes of Brazilian eucalypts wood by natural weathering”, International Wood Products Journal, v. 5, n. 1, pp. 33-38, 2014.

[28] TOLVAJ, L., NÉMETH, K., "Correlation between hue-angle and colour lightness of steamed black locust wood", Acta Silvatica and Lignaria Hungarica, v. 4, pp. 55-59, 2008.

\section{ORCID}

Ezequiel Gallio

Paula Zanatta

Nidria Dias Cruz

Henrique Römer Schulz

Gustavo Spiering Zanol https://orcid.org/0000-0002-0603-1065

https://orcid.org/0000-0003-2200-1189

https://orcid.org/0000-0002-0125-6126

https://orcid.org/0000-0002-8162-7723

https://orcid.org/0000-0002-3244-1447 
